\title{
Statewide Prevalence of Smoke-Free and Vape-Free Homes, by Tobacco Product Use, Minnesota, 2018
}

\author{
Sharrilyn Helgertz, $\mathrm{PhD}^{1}$; Ann St. Claire, $\mathrm{MPH}^{2}$; John Kingsbury, $\mathrm{PhD}^{1}$
}

Accessible Version: www.cdc.gov/pcd/issues/2020/20_0133.htm

Suggested citation for this article: Helgertz S, St. Claire A, Kingsbury J. Statewide Prevalence of Smoke-Free and Vape-Free Homes, by Tobacco Product Use, Minnesota, 2018. Prev Chronic Dis 2020;17:200133. DOI: https://doi.org/10.5888/pcd17.200133.

\section{PEER REVIEWED}

\section{Summary}

What is already known on this topic?

Adults are increasingly making their homes smoke-free and protecting children and other nonsmokers from dangerous secondhand smoke. Ecigarettes pose a new threat to clean indoor air, but few studies have examined whether homes are vape-free.

What is added by this report?

This study provides the first statewide estimate of vape-free rules and shows that most adults who use e-cigarettes, including those who live with children, allow vaping in their homes and are potentially exposing others to secondhand aerosol.

What are the implications for public health practice?

Clean indoor air efforts should promote smoke-free and vape-free homes.

\section{Abstract}

\section{Introduction}

Securing clean indoor air laws is a major tobacco control accomplishment of the past 15 years. The public quickly adopted and supported such policies both in public and private spaces. Clean indoor air is now threatened by the emergence of e-cigarettes. Ecigarette aerosol contains nicotine, heavy metals, and carcinogens, and the long-term effect of secondhand exposure is unknown. Surveillance is necessary to track voluntary rules on smoking and vaping in the home.

\section{Methods}

The Minnesota Adult Tobacco Survey (MATS) is a series of cross-sectional, random-digit-dial telephone surveys on smoking, vaping, and other tobacco-related behaviors, attitudes, and beliefs among Minnesota adults. MATS measured voluntary smoke-free rules in the home in $2014(\mathrm{~N}=9,304)$ and measured both smokefree and vape-free home rules in $2018(\mathrm{~N}=6,055)$.

\section{Results}

The prevalence of smoke-free home rules among Minnesota adults in 2018 was $91.5 \%$ (95\% CI, 90.5\%-92.5\%), up slightly from $89.3 \%(95 \%$ CI, $88.4 \%-90.2 \%)$ in 2014 . In comparison, $84.0 \%$ (95\% CI, 82.7\%-85.3\%) reported vape-free home rules. Although $70.0 \%$ (95\% CI, $66.0 \%-73.0 \%)$ of smokers in 2018 reported smoke-free home rules, only $23.3 \%$ (95\% CI, $15.0 \%-31.6 \%$ ) of ecigarette users reported vape-free home rules. Living with children younger than 18 years significantly increased the odds of having smoke-free and vape-free home rules.

\section{Conclusion}

Although widespread adoption of voluntary smoke-free and vapefree home rules demonstrates a positive change in social norms, most e-cigarette users allow vaping in their homes, including those who live with children younger than 18 . Tracking voluntary smoke-free and vape-free home rules and efforts to encourage them are important to improve the public's health.

\section{Introduction}

Securing clean indoor air laws is a major tobacco control accomplishment of the past 15 years. Still, secondhand tobacco smoke poses a serious health threat to nonsmokers (1). Strong clean indoor air laws have reduced exposure to secondhand smoke in public places (2) and encouraged adoption of smoke-free rules in private spaces $(3,4)$, but the home remains the primary source of secondhand exposure for children and a major source of exposure for nonsmoking adults (1). Although the number of households with smoke-free rules has increased over time (5), adoption of smoke-free home rules is uneven. Households with children are more likely to have rules against smoking in the home; households with adults who are older, have lower income, have less education, or smoke, are less likely $(6,7)$. 
Emerging in 2007, e-cigarettes have been marketed as a healthier alternative to combustible cigarettes, and some advertisements promoted their use where smoking is not allowed (8). E-cigarette aerosol contains nicotine, heavy metals, carcinogens, ultra-fine particulate matter exceeding background levels, and metals, such as nickel and chromium, that exceed levels associated with conventional smoking (9). Although the long-term risks of secondhand aerosol exposure are unknown, studies show that e-cigarette use contaminates the air under controlled (10) and real-world conditions (11), extending the potential health risks beyond the user (12).

Several state and local governments have added e-cigarettes to their smoke-free laws. However, misleading industry marketing, acceptance of e-cigarettes by some public health advocates as a harm-reduction strategy for smokers, and slow regulation by the US Food and Drug Administration have contributed to the public's perception that e-cigarette aerosol is low risk or even harmless (13). Adults who prohibit smoking conventional cigarettes in their homes may allow vaping and unwittingly expose friends and family to potential health risks. Our study uses data from the Minnesota Adult Tobacco Survey (MATS) to describe the first statewide prevalence of vape-free home rules and examine whether e-cigarette use and children in the home predict adoption of vape-free home rules.

\section{Methods}

Data came from the 2014 and 2018 administrations of MATS. MATS is a series of cross-sectional, random-digit-dial landline and cell phone surveys of civilian, noninstitutionalized adults aged 18 or older living in Minnesota. MATS data were collected in 1999, 2003, 2007, 2010, 2014, and 2018. The MATS 2014 final sample included 9,304 respondents; the response rate was $25.2 \%$ for the landline survey and $18.2 \%$ for the cell phone survey. In 2018, MATS included 6,055 participants and yielded American Association for Public Opinion Research (AAPOR) response rates of $17.5 \%$ for the landline survey and $13.4 \%$ for the cell phone survey. The same screening, sampling, and refusal conversion protocols were used in both survey years. Weighting was applied to create unbiased population estimates based on the probability of selection resulting from the sampling plan. Weights were calibrated based on sex, race/ethnicity, location, and education totals from the US Census Bureau's American Community Survey (14). Methodologic details are available at www.clearwaymn.org/MATS. MATS was approved by the Minnesota Department of Health's institutional review board.

\section{Measures}

MATS measured voluntary smoke-free rules in the home in 2014 and 2018 and vape-free rules in 2018. Smoke-free rules were measured by the question, "Which statement best describes rules about smoking inside your home (excluding porches and garages)?" Vape-free rules were measured similarly: "Which statement best describes the rules about using e-cigarettes or vaping devices inside your home (excluding porches and garages)?" Responses included not allowed anywhere, allowed in some places or at some times, or allowed anywhere. Only respondents who indicated that smoking or vaping was not allowed anywhere were considered to have rules against that activity.

Covariates were age (4 categories), sex (male or female), race/ethnicity (5 mutually exclusive categories), education (4 categories), annual household income (4 categories), marital status (married or not), current smoker of cigarettes, cigars, or pipe (yes or no), current e-cigarette user (yes or no), and lives with a child younger than 18 years (yes or no). Respondents who reported currently smoking cigarettes, cigars, or pipe every day or some days and met minimum lifetime-use thresholds (100 cigarettes, 20 times, or 20 times, respectively) were categorized as current smokers. Similarly, respondents who reported currently using e-cigarettes every day or some days were categorized as current e-cigarette users; however, no minimum lifetime use was required.

\section{Analysis}

We performed all analyses using SPSS Statistics version 24 (IBM Corporation) for complex samples. We used pairwise deletion to maximize available data. We used the Pearson $\chi^{2}$ to assess the change from 2014 to 2018 in the percentage of Minnesota adults with smoke-free rules and assess the bivariate association between smoke-free and vape-free rules and respondent characteristics. We used logistic regression to assess the unique association between respondent characteristics and smoke-free rules and vape-free rules in 2018. We entered the entire set of potential predictors simultaneously, testing for main effects only. Ordinal variables were dummy coded. We used odds ratios (ORs) and 95\% CIs to estimate the likelihood of adults reporting smoke-free or vape-free rules.

\section{Results}

The prevalence of smoke-free home rules among Minnesota adults in 2018 was $91.5 \%$ (95\% CI, 90.5\%-92.5\%), up slightly from $89.3 \%(95 \% \mathrm{CI}, 88.4 \%-90.2 \%)$ in $2014\left(\chi_{1}^{2}=21.8 ; P<.001\right)$. Current smokers were less likely than nonsmokers to report hav-

\footnotetext{
The opinions expressed by authors contributing to this journal do not necessarily reflect the opinions of the U.S. Department of Health and Human Services, the Public Health Service, the Centers for Disease Control and Prevention, or the authors' affiliated institutions.
} 
ing smoke-free rules $(70.0 \%$; $95 \% \mathrm{CI}, 66.0 \%-74.0 \%$ vs $96.2 \%$; 95\% CI, 95.6\%-96.8\%). The percentage of smokers with smokefree rules in 2018 was not significantly different from the percentage observed in 2014 (65.5\%; 95\% CI, 63.0\%-68.02\%).

In the bivariate analysis, having smoke-free rules was significantly associated with age, sex, race/ethnicity, education, annual household income, marital status, smoking status, e-cigarette use, and children younger than 18 years in the home (Table 1).

More than 4 in 5 adults $(84.0 \%$; 95\% CI, 82.7\%-85.3\%) reported vape-free home rules in 2018. Having vape-free rules was significantly associated with all respondent characteristics assessed (Table 1). The prevalence of vape-free home rules was considerably lower among adults aged 18 to $24(68.7 \%$; $95 \%$ CI, $63.7 \%-73.7 \%)$ than among adults 65 or older $(93.2 \%$; $95 \% \mathrm{CI}$, $91.9 \%-94.5 \%$ ). Only $23.3 \%$ (95\% CI, $15.0 \%-31.6 \%$ ) of current ecigarette users reported vape-free homes. The percentage of adults who reported vape-free home rules was higher among respondents living with children younger than 18 years $(87.6 \%$; $95 \% \mathrm{CI}$, $85.7 \%-89.5 \%$ ) than among those not living with children younger than 18 years $(81.9 \%$; $95 \% \mathrm{CI}, 80.3 \%-83.5 \%)$.

Association of respondent characteristics with smoke-free rules in the multivariate analysis. When we controlled for other variables in the model, respondent sex, annual household income, and ecigarette use status were no longer associated with smoke-free home rules (Table 2). Adults who were aged 45 to $64(\mathrm{OR}=0.58$; 95\% CI, 0.34-0.99) or Black (OR $=0.46 ; 95 \%$ CI, 0.24-0.88) were less likely to have smoke-free rules than adults aged 18 to 24 or White, respectively. Adults who had a college degree or higher $(\mathrm{OR}=1.99 ; 95 \% \mathrm{CI}, 1.02-3.90)$ or who were married $(\mathrm{OR}=1.66$; 95\% CI, 1.16-2.39) were more likely to have smoke-free rules than adults who had less education or were not married, respectively. Nonsmokers were more likely to have smoke-free rules than smokers (OR $=9.12 ; 95 \% \mathrm{CI}, 6.52-12.76)$. Adults who lived with children younger than 18 years were more likely to report smokefree rules than those not living with children younger than 18 years $(\mathrm{OR}=2.13 ; 95 \% \mathrm{CI}, 1.37-3.32)$. The percentage of smokers who reported smoke-free home rules was greater among those who lived with children $(82.0 \%$; 95\% CI, $76.8 \%-87.2 \%)$ than among those who did not (63.6\%; 95\% CI, 58.5\%-68.7\%).

Association of respondent characteristics with vape-free rules in the multivariate analysis. When we controlled for other variables in the model, sex, race/ethnicity, education, and marital status were no longer associated with vape-free home rules (Table 2). Adults who were older than 25 or had household incomes higher than $\$ 75,000$ were more likely than adults younger than 25 or lower-income adults to report having vape-free rules. Nonsmokers were more likely than smokers to report vape-free rules $(\mathrm{OR}=$
4.73; 95\% CI, 3.61-6.20). Adults who lived with children younger than 18 years were more likely than those not living with children younger than 18 years to report vape-free rules $(\mathrm{OR}=1.87$; $95 \%$ CI, 1.37-2.54). Non-e-cigarette users were more likely than e-cigarette users to report vape-free rules $(\mathrm{OR}=13.76,95 \% \mathrm{CI}$, 8.11-23.36). Less than one-third of adults who used e-cigarettes and lived with children $(29.4 \%$; $95 \% \mathrm{CI}, 13.1 \%-45.7 \%)$ reported vape-free home rules compared to $19.7 \%$ (95\% CI, 10.2\%-29.2\%) of adults who vaped and did not live with children.

\section{Discussion}

The prevalence of smoke-free home rules among Minnesota adults in 2018 was $91.5 \%$, up slightly from $89.3 \%$ in 2014 , and a substantial increase from $64.5 \%$ in 1999 (15). As smoke-free home rules approach universal adoption, persistent disparities in the prevalence of smoke-free home rules are beginning to disappear. In 2018, although older and lower-income adults continued to be less likely to report smoke-free rules than younger or higher-income adults, men and women and adults with varying levels of education were equally likely to report rules against smoking in the home.

However, the emergence of e-cigarettes and the potential for exposure to secondhand aerosol present a new threat to clean indoor air. Our study presents the first statewide estimate of vape-free home rules. In Minnesota, more than 4 in 5 adults (84.0\%) have rules against vaping in the home, but as expected, this percentage was lower than the percentage of adults that have smoke-free home rules $(91.5 \%)$. Although the prevalence of vape-free home rules is moderately high overall, our findings underscore the importance of targeting messages to segments of the population that do not have vape-free home rules. The discrepancy between smoke-free and vape-free rules may be due to the belief by many that electronic cigarettes are less harmful than conventional cigarettes (16). This belief may inappropriately trivialize the risk of secondhand aerosol exposure. A 2017 survey testing public awareness of aerosol constituents showed that $58 \%$ of US adults were unaware that e-cigarette aerosol contains more than water vapor; Black adults and smokers were least likely to have correct knowledge, and correct knowledge was associated with higher perceived harmfulness of secondhand exposure to aerosol (17). Education campaigns warning the public of the potential harms of secondhand aerosol exposure are needed.

Disparities in vape-free rules show marked similarity to historical disparities in smoke-free rules $(6,7,15)$. Our study suggests that having vape-free rules, similar to having smoke-free rules, is largely a function of using the products oneself and having someone in the home to protect from exposure. Adults who use e-

The opinions expressed by authors contributing to this journal do not necessarily reflect the opinions of the U.S. Department of Health and Human Services, the Public Health Service, the Centers for Disease Control and Prevention, or the authors' affiliated institutions. 
cigarettes are less likely to have vape-free rules, whereas adults who live with children younger than 18 years are more likely to prohibit vaping in the home. We observed a stark contrast among those who use these products between allowing smoking and vaping in homes where children are present. Among adults who live with children, $82.0 \%$ of smokers do not smoke in their homes, whereas only $29.4 \%$ of e-cigarette users do not vape in their homes.

Given the popularity of vaping and low prevalence of vape-free home rules among e-cigarette users, the advocacy message should now change from encouraging smoke-free rules to encouraging clean air rules in the home - no smoking or vaping in the home - especially in homes where children live. Smoke-free home rules have played a unique role in discouraging smoking (and increasing quitting [18]) by reducing social acceptance of the behavior and making smoking less convenient, and this phenomenon can likely be extended to vaping. As smoke-free policies in public places are amended to include e-cigarette use and public awareness of the potential harms of secondhand aerosol exposure increases, we anticipate voluntary vape-free rules will also increase. Researchers and public health practitioners can learn from the success of smoke-free efforts and build on existing practice to expedite additional public and private policies and communication campaigns to reduce the harm of secondhand smoke and aerosol exposure. Dedicating resources to successfully educate and promote vape-free home rules will protect nonusers from aerosol exposure and potentially contribute to youth vaping prevention. To inform intervention efforts, it would be helpful if future research explored the characteristics of people who allow vaping in their homes, especially those who allow vaping but not smoking, because this group may be particularly responsive to education on the risks of secondhand aerosol.

Our study has limitations. MATS did not assess the perceived harmfulness of secondhand aerosol or whether others in the respondent's household vape, so we could not include these variables - which are likely to be associated with vape-free rules in our analysis. Our study relies on self-report, and self-reported data are subject to some degree of social desirability and recall biases. Our data are from a state with strong antismoking norms and policies, so the findings may not generalize nationally or to other states.

Although widespread adoption of voluntary smoke-free and vapefree home rules demonstrates positive social norm change, most ecigarette users in our study allowed vaping in their homes, including those who live with children younger than 18 years. Tracking voluntary smoke-free and vape-free home rules is an important component of tobacco control and demonstrates where resources should be directed to improve the public's health.

\section{Acknowledgments}

The authors thank Michael Parks and Raymond Boyle for helpful comments on earlier drafts of this manuscript. No financial support was provided for the preparation of this manuscript. No copyrighted materials were used in this research.

\section{Author Information}

Corresponding Author: Sharrilyn Helgertz, PhD, Senior Research Scientist, Minnesota Department of Health, Center for Health Statistics, 85 East 7th Pl, PO Box 64882, Saint Paul, MN 551640882 . Te le phone: $651-201-5942$. E mai 1: sharrilyn.helgertz@state.mn.us.

Author Affiliations: ${ }^{1}$ Minnesota Department of Health, St Paul, Minnesota. ${ }^{2}$ ClearWay Minnesota, Minneapolis, Minnesota.

\section{References}

1. US Department of Health and Human Services. The health consequences of involuntary exposure to tobacco smoke: a report of the Surgeon General. Atlanta (GA): US Department of Health and Human Services, Centers for Disease Control and Prevention, Coordinating Center for Health Promotion, National Center for Chronic Disease Prevention and Health Promotion, Office on Smoking and Health; 2006.

2. Eriksen MP, Cerak RL. The diffusion and impact of clean indoor air laws. Annu Rev Public Health 2008;29(1):171-85.

3. Cheng KW, Okechukwu CA, McMillen R, Glantz SA. Association between clean indoor air laws and voluntary smokefree rules in homes and cars. Tob Control 2015; 24(2):168-74.

4. Cheng K-W, Glantz SA, Lightwood JM. Association between smokefree laws and voluntary smokefree-home rules. Am J Prev Med 2011;41(6):566-72.

5. King BA, Patel R, Babb SD, Hartman AM, Freeman A. National and state prevalence of smoke-free rules in homes with and without children and smokers: two decades of progress. Prev Med 2016;82:51-8.

6.Zhang X, Martinez-Donate AP, Kuo D, Jones NR, Palmersheim KA. Trends in home smoking bans in the U.S.A., 1995-2007: prevalence, discrepancies and disparities. Tob Control 2012;21(3):330-6.

7. Parks MJ, Kingsbury JH, Boyle RG, Evered S. Smoke-free rules in homes and cars among smokers and nonsmokers in Minnesota. Prev Chronic Dis 2018;15:E32.

The opinions expressed by authors contributing to this journal do not necessarily reflect the opinions of the U.S. Department of Health and Human Services, the Public Health Service, the Centers for Disease Control and Prevention, or the authors' affiliated institutions. 
8. US Department of Health and Human Services. E-cigarette use among youth and young adults. a report of the Surgeon General. Atlanta (GA): US Department of Health and Human Services, Centers for Disease Control and Prevention, National Center for Chronic Disease Prevention and Health Promotion, Office on Smoking and Health; 2016.

9. Wilson N, Hoek J, Thomson G, Edwards R. Should e-cigarette use be included in indoor smoking bans? Bull World Health Organ 2017;95(7):540-1.

10. Schripp T, Markewitz D, Uhde E, Salthammer T. Does ecigarette consumption cause passive vaping? Indoor Air 2013; 23(1):25-31.

11. Chen R, Aherrera A, Isichei C, Olmedo P, Jarmul S, Cohen JE, et al. Assessment of indoor air quality at an electronic cigarette (Vaping) convention. J Expo Sci Environ Epidemiol 2018; 28(6):522-9.

12. Hess IM, Lachireddy K, Capon A. A systematic review of the health risks from passive exposure to electronic cigarette vapour. Public Health Res Pract 2016;26(2):e2621617.

13. Unger JB, Escobedo P, Allem J-P, Soto DW, Chu K-H, Cruz T. Perceptions of secondhand e-cigarette aerosol among Twitter users. Tob Regul Sci 2016;2(2):146-52.

14. US Census Bureau. American Community Survey (ACS). https://www.census.gov/programs-surveys/acs. Accessed October 7, 2020.

15. St Claire AW, Boyle RG, Schillo BA, Rode P, Taylor KA. Smokefree home rules adoption by smokers and nonsmokers: Minnesota, 1999-2010. Am J Prev Med 2012;43(5Suppl 3):S197-204.

16. Huang J, Feng B, Weaver SR, Pechacek TF, Slovic P, Eriksen MP. Changing perceptions of harm of e-cigarette vs cigarette use among adults in 2 US national surveys from 2012 to 2017. JAMA Netw Open 2019;2(3):e191047.

17. Tan ASL, Mello S, Sanders-Jackson A, Bigman CA. Knowledge about chemicals in e-cigarette secondhand vapor and perceived harms of exposure among a National Sample of U.S. adults. Risk Anal 2017;37(6):1170-80.

18. Hyland A, Higbee C, Travers MJ, Van Deusen A, BansalTravers M, King B, et al. Smoke-free homes and smoking cessation and relapse in a longitudinal population of adults. Nicotine Tob Res 2009;11(6):614-8.

The opinions expressed by authors contributing to this journal do not necessarily reflect the opinions of the U.S. Department of Health and Human Services, the Public Health Service, the Centers for Disease Control and Prevention, or the authors' affiliated institutions. 


\section{Tables}

Table 1. Prevalence of Smoke-Free Rules and Vape-Free Rules Among Minnesota Adults, Minnesota Adult Tobacco Survey, 2018

\begin{tabular}{|c|c|c|c|}
\hline Characteristic & Unweighted No. (\%) ${ }^{a}$ & Smoke-Free Home, \% (95\% Cl) & Vape-Free Home, $\%(95 \% \mathrm{Cl})^{\mathrm{b}}$ \\
\hline Overall & $5,538(100.0)$ & $91.5(90.5-92.5)$ & $84.0(82.7-85.3)$ \\
\hline \multicolumn{4}{|l|}{ Age, $y$} \\
\hline $18-24$ & $421(7.6)$ & $91.1(88.5-93.7)$ & $68.7(63.7-73.7)$ \\
\hline $25-44$ & $1,318(23.8)$ & $93.4(91.9-94.9)$ & $83.1(80.9-85.3)$ \\
\hline $45-64$ & $1,950(35.2)$ & $89.4(87.6-91.2)$ & $85.4(83.4-87.4)$ \\
\hline$\geq 65$ & $1,849(33.4)$ & $92.3(90.8-93.8)$ & $93.2(91.9-94.5)$ \\
\hline \multicolumn{4}{|l|}{ Sex } \\
\hline Male & $2,569(46.4)$ & $90.2(88.8-91.6)$ & $81.6(79.7-83.5)$ \\
\hline Female & $2,969(53.6)$ & $92.8(91.5-94.1)$ & $86.3(84.7-87.9)$ \\
\hline \multicolumn{4}{|l|}{ Race/ethnicity } \\
\hline Non-Hispanic White & $4,550(82.2)$ & $92.0(91.0-93.0)$ & $84.4(83.0-85.8)$ \\
\hline Hispanic & $271(4.9)$ & $94.5(91.6-97.4)$ & $85.0(79.4-90.6)$ \\
\hline Multi/other & $254(4.6)$ & $79.9(70.6-89.2)$ & $64.8(55.3-74.3)$ \\
\hline Non-Hispanic Black & $239(4.3)$ & $84.9(79.6-90.2)$ & $83.8(78.5-89.1)$ \\
\hline Asian & $153(2.8)$ & $94.5(91.7-97.3)$ & $87.7(82.7-92.7)$ \\
\hline \multicolumn{4}{|l|}{ Education } \\
\hline$<$ High school graduate & $182(3.3)$ & $82.5(76.4-88.6)$ & $71.6(64.0-79.2)$ \\
\hline High school graduate/GED & $1,180(21.3)$ & $87.3(85.2-89.4)$ & $82.8(80.4-85.2)$ \\
\hline Some college or technical school & $1,797(32.4)$ & $91.3(89.8-92.8)$ & $80.6(78.2-83.0)$ \\
\hline$\geq$ College graduate & $2,346(42.4)$ & $97.0(96.3-97.7)$ & $91.3(90.0-92.6)$ \\
\hline \multicolumn{4}{|l|}{ Annual household income, $\$$} \\
\hline$\leq 35,000$ & $1,142(20.6)$ & $83.9(81.0-86.8)$ & $74.5(71.0-78.0)$ \\
\hline $35,001-50,000$ & $590(10.7)$ & $89.7(87.1-92.3)$ & $82.9(79.3-86.5)$ \\
\hline $50,001-75,000$ & $878(15.9)$ & $92.3(90.1-94.5)$ & $84.8(81.8-87.8)$ \\
\hline$\geq 75,001$ & $2,247(40.6)$ & $95.4(94.3-96.5)$ & $88.1(86.4-89.8)$ \\
\hline \multicolumn{4}{|l|}{ Marital status } \\
\hline Married & $2,896(52.3)$ & 94.9 (93.9-95.9) & $90.6(89.3-91.9)$ \\
\hline Not married & $2,617(47.3)$ & $87.5(85.8-89.2)$ & $76.2(73.9-78.5)$ \\
\hline \multicolumn{4}{|c|}{ Current smoker of cigarettes, cigars, or pipe } \\
\hline Yes & $765(13.8)$ & $70.0(66.0-74.0)$ & $55.8(51.3-60.3)$ \\
\hline No & $4,752(85.8)$ & $96.2(95.6-96.8)$ & $90.2(89.0-91.4)$ \\
\hline \multicolumn{4}{|l|}{ Current e-cigarette user } \\
\hline Yes, every/some days & $178(3.2)$ & $80.1(73.9-86.3)$ & $23.3(15.0-31.6)$ \\
\hline
\end{tabular}

Abbreviation: GED, general equivalency diploma.

${ }^{a}$ Percentages are based on the value in the column heading; some percentages may not sum to 100 because of missing data (respondents declined to answer question or responded with "don't know").

${ }^{\mathrm{b}}$ All Pearson $\mathrm{x}^{2}$ tests of independence between smoke-free and vape-free home rules were significant at $P<.01$. 


\section{PREVENTING CHRONIC DISEASE}

VOLUME 17, E141

PUBLIC HEALTH RESEARCH, PRACTICE, AND POLICY

(continued)

Table 1. Prevalence of Smoke-Free Rules and Vape-Free Rules Among Minnesota Adults, Minnesota Adult Tobacco Survey, 2018

\begin{tabular}{|l|c|c|c|}
\hline Characteristic & Unweighted No. (\%) & Smoke-Free Home, \% (95\% Cl) $^{\mathbf{b}}$ & Vape-Free Home, \% (95\% Cl) ${ }^{\mathbf{b}}$ \\
\hline No, not at all & $5,359(96.8)$ & $92.1(91.2-93.0)$ & $87.0(85.8-88.2)$ \\
\hline Children in the home & $1,486(26.8)$ & $95.2(94.0-96.4)$ & $87.6(85.7-89.5)$ \\
\hline Yes & $4,048(73.1)$ & $89.4(88.0-90.8)$ & $81.9(80.3-83.5)$ \\
\hline No &
\end{tabular}

Abbreviation: GED, general equivalency diploma.

${ }^{a}$ Percentages are based on the value in the column heading; some percentages may not sum to 100 because of missing data (respondents declined to answer question or responded with "don't know").

${ }^{b}$ All Pearson $x^{2}$ tests of independence between smoke-free and vape-free home rules were significant at $P<.01$. 
Table 2. Adjusted and Weighted Odds of Smoke-Free and Vape-Free Rules Among Minnesota Adults, Minnesota Adult Tobacco Survey, 2018

\begin{tabular}{|c|c|c|}
\hline Characteristics & Have Smoke-Free Rules, Odds Ratio (95\% Cl) & Have Vape-Free Rules, Odds Ratio $(95 \% \mathrm{Cl})$ \\
\hline \multicolumn{3}{|l|}{ Age, $y$} \\
\hline $18-24$ & 1 [Reference] & 1 [Reference] \\
\hline $25-44$ & $1.16(0.66-2.04)$ & $1.53(1.03-2.26)^{a}$ \\
\hline $45-64$ & $0.58(0.34-0.99)^{a}$ & $1.80(1.21-2.67)^{a}$ \\
\hline$\geq 65$ & $0.62(0.35-1.10)$ & $3.54(2.23-5.60)^{a}$ \\
\hline \multicolumn{3}{|l|}{ Sex } \\
\hline Male & 1 [Reference] & 1 [Reference] \\
\hline Female & $1.23(0.90-1.69)$ & $1.17(0.92-1.50)$ \\
\hline \multicolumn{3}{|l|}{ Race/ethnicity } \\
\hline White & 1 [Reference] & 1 [Reference] \\
\hline Hispanic & $1.27(0.52-3.09)$ & $1.23(0.65-2.31)$ \\
\hline Multi/other & $0.55(0.23-1.29)$ & $0.57(0.30-1.05)$ \\
\hline Black & $0.46(0.24-0.88)^{a}$ & $1.16(0.68-1.99)$ \\
\hline Asian & $0.61(0.24-1.51)$ & $1.11(0.52-2.38)$ \\
\hline \multicolumn{3}{|l|}{ Education } \\
\hline$<$ High school graduate & 1 [Reference] & 1 [Reference] \\
\hline High school graduate/GED & $0.88(0.48-1.62)$ & $1.18(0.67-2.09)$ \\
\hline Some college or technical school & $1.14(0.61-2.12)$ & $0.86(0.49-1.50)$ \\
\hline$\geq$ College graduate & $1.99(1.02-3.90)^{a}$ & $1.30(0.73-2.33)$ \\
\hline \multicolumn{3}{|l|}{ Annual household income, $\$$} \\
\hline$\leq 35,000$ & 1 [Reference] & 1 [Reference] \\
\hline $35,001-50,000$ & $1.15(0.72-1.84)$ & $1.44(0.98-2.13)$ \\
\hline $50,001-75,000$ & $1.17(0.72-1.90)$ & $1.40(0.95-2.07)$ \\
\hline$\geq 75,001$ & $1.58(0.99-2.52)$ & $1.60(1.13-2.26)^{a}$ \\
\hline \multicolumn{3}{|l|}{ Marital status } \\
\hline Married & $1.66(1.16-2.39)^{a}$ & $1.31(0.99-1.74)$ \\
\hline Not married & 1 [Reference] & 1 [Reference] \\
\hline \multicolumn{3}{|c|}{ Current smoker of cigarettes, cigars, or pipe } \\
\hline Yes & 1 [Reference] & 1 [Reference] \\
\hline No & $9.12(6.52-12.76)^{a}$ & $4.73(3.61-6.20)$ \\
\hline \multicolumn{3}{|l|}{ Current e-cigarette user } \\
\hline Yes, every/some days & 1 [Reference] & 1 [Reference] \\
\hline No, not at all & $1.41(0.78-2.56)$ & $13.76(8.11-23.36)^{a}$ \\
\hline \multicolumn{3}{|l|}{ Children in the home } \\
\hline Yes & $2.13(1.37-3.32)^{a}$ & $1.87(1.37-2.54)^{a}$ \\
\hline No & 1 [Reference] & 1 [Reference] \\
\hline
\end{tabular}

a Significant at $P<.05$.

The opinions expressed by authors contributing to this journal do not necessarily reflect the opinions of the U.S. Department of Health and Human Services, the Public Health Service, the Centers for Disease Control and Prevention, or the authors' affiliated institutions. 